\title{
Acute Effects of Two Angiotensin Receptor Blockers on Vascular Hemodynamics, Arterial Stiffness, and Oxidative Stress in Patients with Mild to Moderate Hypertension: An Open Label Parallel Group Study
}

\author{
Rama Mohan Pathapati, ${ }^{1}$ Meriga Rajesh Kumar, ${ }^{1}$ Bhakthavatsala Reddy Chirra, ${ }^{1}$ \\ Madhavulu Buchineni, ${ }^{1}$ Sujith TR, ${ }^{1}$ Sreebhushan Raju Devaraju, ${ }^{2}$ and M. U. R. Naidu ${ }^{2}$ \\ ${ }^{1}$ Narayana Medical College and Hospital, Chinthareddypalem, Nellore, Andhra Pradesh 524002, India \\ ${ }^{2}$ Nizams Institute of Medical Sciences, Panjagutta, Hyderabad, Andhra Pradesh 500082, India \\ Correspondence should be addressed to Rama Mohan Pathapati; pill4ill@yahoo.co.in
}

Received 1 November 2012; Accepted 9 December 2012

Academic Editors: A. Mugge and P. Schoenhagen

Copyright (C) 2013 Rama Mohan Pathapati et al. This is an open access article distributed under the Creative Commons Attribution License, which permits unrestricted use, distribution, and reproduction in any medium, provided the original work is properly cited.

Introduction. We studied the acute effects of Olmesartan and Telmisartan at baseline and at the end of four weeks on indices of hemodynamics (heart rate HR, blood pressure BP), vascular (carotid femoral pulse wave velocity cf PWV, digital arterial tone expressed as Reflection index RI, and endothelial dependent vasodilator response EDVR), and oxidative stress (serum Malondialdehyde MDA) in hypertensive patients. Materials and Methods. The eligible patients were randomly allocated to either $20 \mathrm{mg}$ Olmesartan or $40 \mathrm{mg}$ Telmisartan. Results. 40 subjects received Olmesartan, and 29 received Telmisartan. After four weeks of treatment the mean changes from baseline in the Olmesartan group versus Telmisartan group are SBP $-9.8 \pm 10$ versus $-6.3 \pm 12 \mathrm{~mm}$ $\mathrm{Hg}, P=0.24 ; \mathrm{DBP}-6.1 \pm 11$ versus $-4.2 \pm 12.5 \mathrm{~mm} \mathrm{Hg}, P=0.55 ; \mathrm{cf} \mathrm{PWV}-1.6 \pm 1.2$ versus $-0.9 \pm 1.4 \mathrm{~m} / \mathrm{s}, P=0.04 ; \mathrm{EDVR}-8.2 \pm 5.2$ versus $-5.2 \pm 5.7 \%, P=0.04$; and $\mathrm{MDA}-1.9 \pm 1.1$ versus $-1.2 \pm 1.2 \eta \mathrm{Mol} / \mathrm{mL}, P=0.03$. Conclusion. Olmesartan showed a better improvement in cf PWV, EDVR, and MDA than Telmisartan with an identical reduction in blood pressure.

\section{Introduction}

In hypertension, whole arterial system undergoes both structural and functional alterations. The alteration in function occurs early and manifests as decreased endothelial dependent vasodilator response and increased arterial stiffness. It can be understood that one or every possible mechanism such as an imbalance between L-arginine-nitric oxide system, mechanical stretch due to high blood pressure, oxidative stress, and endothelial dysfunction may be liable for increased resting arterial tone [1-6]. Angiotensin receptor blockers (ARB) may offer potential therapeutic benefits on cardiovascular system through multiple mechanisms, in addition to blood pressure lowering effect. However, selective inhibition of the AT-II type 1 depends on the affinity of the ARB to the receptor. Olmesartan and Telmisartan have high binding affinities to type- 1 receptors than other ARBs. To this purpose, we studied the acute effects of Olmesartan and Telmisartan on indices of hemodynamics, (Heart rate HR, Blood pressure BP), vascular (carotid femoral pulse wave velocity cf PWV, digital arterial tone expressed as Reflection index RI, and endothelial dependent vasodilator response EDVR), and oxidative stress (serum Malondialdehyde MDA) in hypertensive patients.

\section{Materials and Methods}

The Institutional Ethical Committee approved this open label randomized parallel group study protocol. Informed consent was obtained from study participants. All the treatment naive patients in the age group between 20 to 60 years with sitting systolic blood pressure between $140-160 \mathrm{mmHg}$ and diastolic blood pressure between 90-99 Hg were included in 
the study. Patients were excluded if the hypertension was secondary to hepatic, renal, cardiac, or endocrine disorders; pregnant or lactating; receiving Olmesartan or Telmisartan; or hypersensitive to Salbutamol, Olmesartan, or Telmisartan. The eligible patients randomly received either $20 \mathrm{mg}$ of Olmesartan or $40 \mathrm{mg}$ of Telmisartan. The treatments were given for duration of four weeks. BP, HR, cf PWV, RI, EDVR, and MDA were measured at baseline and at the end of four weeks. Demographic, clinical, and laboratory records of the enrolled patients were also recorded. After the completion of the study, the patients were instructed to consult their physician for further management.

2.1. Pulse Wave Velocity. The cf PWV was measured using a volume-plethysmographic apparatus (Periscope, M/S Geneisis Medical Systems, Hyderabad). This instrument also records blood pressure and electrocardiogram. The subjects were examined in the spine position after a 10-minute rest, with electrodes connected to all the four limbs and cuffs wrapped on both the brachia and ankles. The plethysmographic sensor and the oscillometric pressure sensors were positioned in these cuffs records volume waveforms and blood pressure, respectively. Initially the brachial ankle pulse wave velocities baPWV of the right and left were obtained from the stored wave forms. Subsequently, cf PWV was calculated automatically from the mathematical equation of $\mathrm{PWV}=0.833^{*}$ average baPWV-233.3 [7].

2.2. Endothelial Dependent Vasodilatory Function. RI was measured from the waveforms recorded from the index finger of the right hand by digital volume plethysmography (Dicrowin, M/S Genesis Medical Systems, Hyderabad) as per the procedure described in detail by Naidu et al. [8]. At each visit RI was measured at base line, then 400 micrograms of Salbutamol was administered through spacer, and at the end of 15 minutes after inhalation RI was measured again. At each time, three such recordings were collected. The mean of these three measurements was used for final analysis. The percentage decrease in RI at the end of the test compared to baseline was used to assess EDVR [9].

2.3. Serum MDA (Malondialdehyde). Measurement of MDA provides a sensitive index of the lipid peroxidation. The lipid peroxidation products react with thiobarbituric acid forming a pink coloured adduct on boiling. At each visit, $6 \mathrm{~mL}$ of blood was collected in clean dry test tubes. The test tubes were then centrifuged at $2000 \mathrm{rpm}$ for 10 minutes; serum was separated and collected in aliquots labeled with patient code and date of collection and they were stored at $20^{\circ}$ centigrade in a refrigerator for the analysis of serum MDA. To $0.5 \mathrm{~mL}$ of serum, $0.5 \mathrm{~mL}$ of $20 \%$ TCA (trichloroacetic acid) and 250 microliters of TBA reagent $(0.33 \%$, Sigma Chemical Co., USA) were added in plastic tubes. These tubes were first kept in water bath for 60 minutes at 95 degrees centigrade then cooled and vortexed. The absorbance of the supernatant was read at $532 \mathrm{~nm}$ at room temperature against blank. The concentration of MDA was read from standard calibration
TABLE 1: Base line clinical, hemodynamic, and vascular characteristics of mild-moderate essential hypertensive patients according to treatment group.

\begin{tabular}{lccc}
\hline Parameter & $\begin{array}{c}\text { Olmesartan } \\
(n=40)\end{array}$ & $\begin{array}{c}\text { Telmisartan } \\
(n=29)\end{array}$ & $P$ value \\
\hline Age, y & $47 \pm 11$ & $42 \pm 14$ & 0.34 \\
Gender, M/F & $29 / 11$ & $20 / 9$ & 0.24 \\
Height, cm & $162.57 \pm 18.1$ & $164.82 \pm 18.3$ & 0.76 \\
Weight, Kg & $66.82 \pm 10.8$ & $65.48 \pm 9.6$ & 0.75 \\
Body mass index, g/m ${ }^{2}$ & $25.8 \pm 2.2$ & $24.3 \pm 3.2$ & 0.22 \\
Median duration of & & & \\
hypertension & $3.26(1.2-5.6)$ & $4.24(2.1-5.6)$ & 0.38 \\
(range) months & & & \\
Diabetes & 12 & 10 & 0.79 \\
Current smoker & 8 & 7 & 0.77 \\
Alcoholics & 5 & 4 & 1.0 \\
Lipid-lowering drug & 13 & 9 & 1.0 \\
Serum creatinine, mg/dL & $0.88 \pm 0.18$ & $0.91 \pm 0.16$ & 0.67 \\
Random blood glucose, & $92.1 \pm 10.3$ & $96.7 \pm 8.5$ & 0.09 \\
mg/dL & & & \\
Total cholesterol, mg/dL & $207.2 \pm 23.0$ & $216.5 \pm 32.3$ & 0.17 \\
HDL cholesterol, mg/dL & $55.8 \pm 14.7$ & $51.9 \pm 19.9$ & 0.31 \\
LDL cholesterol, mg/dL & $127.1 \pm 28.1$ & $135.9 \pm 42.9$ & 0.34 \\
MDA, $\eta$ mol/mL & $5.8 \pm 2.8$ & $6.4 \pm 3.0$ & 0.34 \\
HR, beats/min & $80.6 \pm 8.2$ & $76.5 \pm 5.5$ & 0.07 \\
SBP, mm Hg & $146.9 \pm 9.1$ & $147.8 \pm 11.4$ & 0.74 \\
MBP, mm Hg & $112.9 \pm 12.1$ & $116.1 \pm 12.4$ & 0.63 \\
DBP, mm Hg & $95.9 \pm 10.4$ & $93.8 \pm 11.8$ & 0.48 \\
PP, mm Hg & $52.3 \pm 6.2$ & $55.7 \pm 7.4$ & 0.06 \\
cf PWV, m/s & $11.34 \pm 1.4$ & $12.1 \pm 2.1$ & 0.15 \\
RI, \% & $64.6 \pm 9.9$ & $67.6 \pm 9.6$ & 0.26
\end{tabular}

Absolute change at the end of treatment

Data are expressed as mean \pm SD. HDL indicates high-density lipoprotein, LDL: low-density lipoprotein, MDA: plasma Malondialdehyde, SBP: systolic blood pressure, MBP: mean blood pressure, DBP: diastolic blood pressure, PP: pulse pressure, cf PWV: carotid femoral pulse wave velocity, RI: Reflection index.

curve plotted using 1,1,3,3-tetra ethoxy propane. The results were presented in nanomoles of MDA per mL of serum [10].

2.4. Statistical Evaluation. The data was entered in a Microsoft Excel 2003 spreadsheet. The statistical analysis was conducted by means of Sigma GraphPad Prism software version 4, USA. Continuous data was presented as mean, median, range, and standard deviation. Categorical data was presented as actual numbers and percentages. For normally distributed data, within group analysis was performed by using paired $t$ test and between group analyses by unpaired $t$ test. Nonnormally distributed data was analyzed by using nonparametric Mann-Whitney $U$ test. Categorical variables were analyzed with Fischer's exact test. All the efficacy parameters were presented as absolute change from base 
TABLE 2: Comparison of hemodynamic, vascular, and oxidative stress parameters before and after Olmesartan and Telmisartan in patients with hypertension.

\begin{tabular}{|c|c|c|c|c|c|c|}
\hline \multirow{2}{*}{ Parameter } & \multicolumn{3}{|c|}{ Olmesartan $(n=40)$} & \multicolumn{3}{|c|}{ Telmisartan $(n=29)$} \\
\hline & Pre & Post & $P$ value & Pre & Post & $P$ value \\
\hline $\mathrm{HR}$, beats/min & $80.6 \pm 8.2$ & $83.2 \pm 7.1$ & 0.06 & $76.5 \pm 5.5$ & $78.3 \pm 4.5$ & 0.08 \\
\hline SBP, mm Hg & $146.9 \pm 9.1$ & $137.9 \pm 10.8$ & 0.0001 & $147.8 \pm 11.4$ & $134.2 \pm 13.4$ & 0.001 \\
\hline MBP, mm Hg & $112.9 \pm 12.1$ & $100.7 \pm 12.6$ & 0.0001 & $116.1 \pm 12.4$ & $103.2 \pm 11.8$ & 0.022 \\
\hline DBP, mm Hg & $95.9 \pm 10.4$ & $85.6 \pm 9.7$ & $<0.0001$ & $93.8 \pm 11.8$ & $82.2 \pm 11.1$ & 0.003 \\
\hline PP, mm Hg & $52.3 \pm 6.2$ & $46.3 \pm 7.3$ & 0.0002 & $55.7 \pm 7.4$ & $47.3 \pm 8.2$ & 0.002 \\
\hline cf PWV, m/s & $11.34 \pm 1.4$ & $10.07 \pm 1.7$ & 0.0005 & $12.1 \pm 2.1$ & $10.38 \pm 1.9$ & 0.02 \\
\hline RI, \% & $64.6 \pm 9.9$ & $57.7 \pm 11.9$ & 0.006 & $67.6 \pm 9.6$ & $61.4 \pm 12.9$ & 0.09 \\
\hline EDVR, \% & $-3.23 \pm 6.9$ & $-9.02 \pm 8.03$ & 0.006 & $-4.0 \pm 7.1$ & $-8.69 \pm 7.4$ & 0.047 \\
\hline $\mathrm{MDA}, \eta \mathrm{mol} / \mathrm{mL}$ & $5.8 \pm 2.8$ & $4.4 \pm 2.4$ & 0.02 & $6.4 \pm 2.8$ & $5.1 \pm 2.9$ & 0.044 \\
\hline
\end{tabular}

HR: heart rate, SBP: systolic blood pressure, MBP: mean blood pressure, DBP: diastolic blood pressure, PP: pulse pressure, cf PWV: carotid femoral pulse wave velocity, RI: Reflection index, EDVR: endothelial dependent vascular response to salbutamol challenge, MDA: serum malondialdehyde.

line. Negative sign indicates decrease and vice versa. For statistical significance, a two-tailed probability value of less than 0.05 was considered. A sample of 25 patients per group was required to demonstrate an estimated change in primary efficacy variable cf PWV of $1 \mathrm{~m} / \mathrm{s}$ from baseline in both groups, with $80 \%$ power to detect the difference and twosided alpha error of 0.05 . Additionally 30 percent of subjects were added to each group in view of dropouts and missing data. A total of 40 subjects have been planned to allocate to each treatment.

\section{Results}

Sixty-nine subjects participated in the study, 40 received Olmesartan, and 29 received Telmisartan. There were 29 males and 11 females in the Olmesartan group and 20 males and 9 females in the Telmisartan group. They were age and sex matched and the demographic details were given in Table 1. The baseline hemodynamic and vascular parameters presented in Table 2 did not significantly differ between the two groups. The vascular parameters between Olmesartan group versus Telmisartan groups were cf PWV $11.34 \pm 1.4 \mathrm{~m} / \mathrm{sec}$ versus $12 \pm 2.1 \mathrm{~m} / \mathrm{sec}, P=0.15$ (Figure 1 ) and RI $64.6 \pm 9.9 \%$ versus $67.6 \pm 9.6 \%, P=0.26$. The test applied to identify EDVR was also identical in Olmesartan $(-3.23 \pm 6.9 \%)$ and Telmisartan groups $(-4.0 \pm 7.1 \%, P=0.67)$ before the treatment (Figures 2 and 3 ).

It can be seen from Table 3, the mean changes from baseline in the hemodynamic parameters in the Olmesartan group versus Telmisartan group are $\mathrm{HR}+3.3 \pm 7.7$ versus $+2 \pm 6.1$ beats $/ \mathrm{min}, P=0.51$; SBP $-9.8 \pm 10$ versus $-6.3 \pm$ $12 \mathrm{mmHg}, P=0.24$; DBP $-6.1 \pm 11$ versus $-4.2 \pm 12.5 \mathrm{mmHg}$, $P=0.55$, with no statistically significant difference between the two groups. We observed a statistically significant decline in the central artery stiffness in the Olmesartan group as compared to Telmisartan group, cf PWV $-1.6 \pm 1.2$ versus $-0.9 \pm 1.4 \mathrm{~m} / \mathrm{s} P=0.04$, but not in the peripheral digital artery tone, RI $-1.8 \pm 14.6$ versus $-2.4 \pm 12.8 \% P=0.87$. The EDVR was statistically prominent in Olmesartan group as compared Telmisartan group, $-8.2 \pm 5.2$ versus $-5.2 \pm 5.7 \%, P=0.04$.
TABLE 3: Comparison of mean change from baseline in the indices hemodynamic, vascular and lipid peroxidation between Olmesartan and Telmisartan groups.

\begin{tabular}{lccc}
\hline Parameters & $\begin{array}{c}\text { Olmesartan } \\
(n=40)\end{array}$ & $\begin{array}{c}\text { Telmisartan } \\
(n=29)\end{array}$ & $P$ value \\
\hline HR, beats/min & $+3.3 \pm 7.7$ & $+2 \pm 6.1$ & 0.51 \\
SBP, mm Hg & $-9.8 \pm 10$ & $-6.3 \pm 12$ & 0.24 \\
MBP, mm Hg & $-8.5 \pm 8.4$ & $-9.9 \pm 7.1$ & 0.45 \\
DBP, mm Hg & $-6.1 \pm 11$ & $-4.2 \pm 12.5$ & 0.55 \\
PP, mm Hg & $-5.3 \pm 9.5$ & $-3.6 \pm 11.4$ & 0.54 \\
cf PWV, m/s & $-1.6 \pm 1.2$ & $-0.9 \pm 1.4$ & 0.04 \\
RI, \% & $-1.8 \pm 14.6$ & $-2.4 \pm 12.8$ & 0.87 \\
EDVR, \% & $-8.2 \pm 5.2$ & $-5.2 \pm 5.7$ & 0.04 \\
MDA, $\eta \mathrm{mol} / \mathrm{mL}$ & $-1.9 \pm 1.1$ & $-1.2 \pm 1.2$ & 0.03 \\
\hline
\end{tabular}

HR: heart rate, SBP: systolic blood pressure, MBP: mean blood pressure, DBP: diastolic blood pressure, PP: pulse pressure, cf PWV: carotid femoral pulse wave velocity, RI: Reflection index, EDVR: endothelial dependent vascular response to Salbutamol challenge, MDA: serum Malondialdehyde.

This observation was complemented by a significant decrease in the marker of oxidative stress in the Olmesartan group as compared to Telmisartan group, MDA $-1.9 \pm 1.1$ versus $-1.2 \pm$ $1.2 \eta \mathrm{Mol} / \mathrm{mL}, P=0.03$. All the subjects who participated in the study showed good compliance and none of them developed side effects.

\section{Discussion}

In this study, treatment with ARB Olmesartan and Telmisartan resulted in significant decrease in RI and PWV from baseline in hypertensive patients. This reduction in aortic PWV and arterial wave reflections was in parallel to the decrease of blood pressure. It is possible that such an improvement in arterial compliance in our study may involve acute functional changes such as vascular smooth muscle relaxation and blood pressure reduction. Beuge and Aust [11] reported that treatment with Losartan for 4 weeks significantly reduced PWV with BP reduction. Mahmud and Feely [12] reported that in 


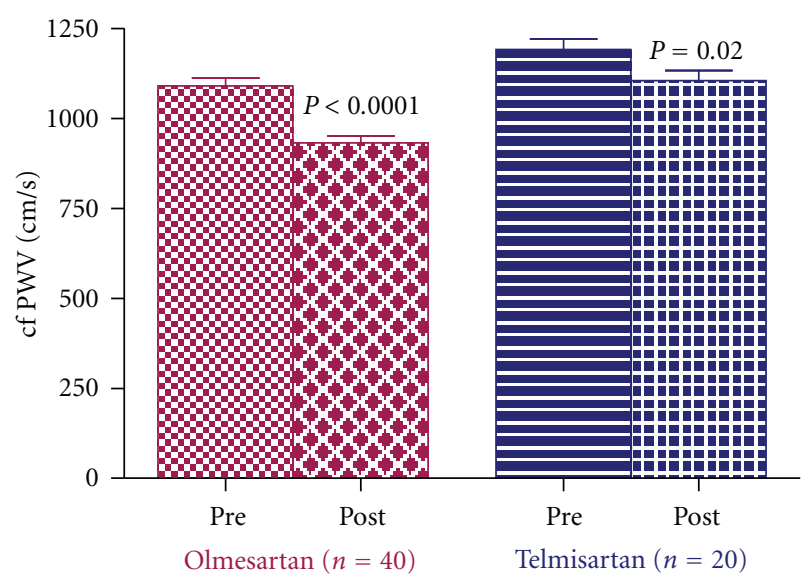

Figure 1: Effect of Olmesartan versus Telmisartan on carotid femoral pulse wave velocity (cf PWV) in patients with Hypertension.

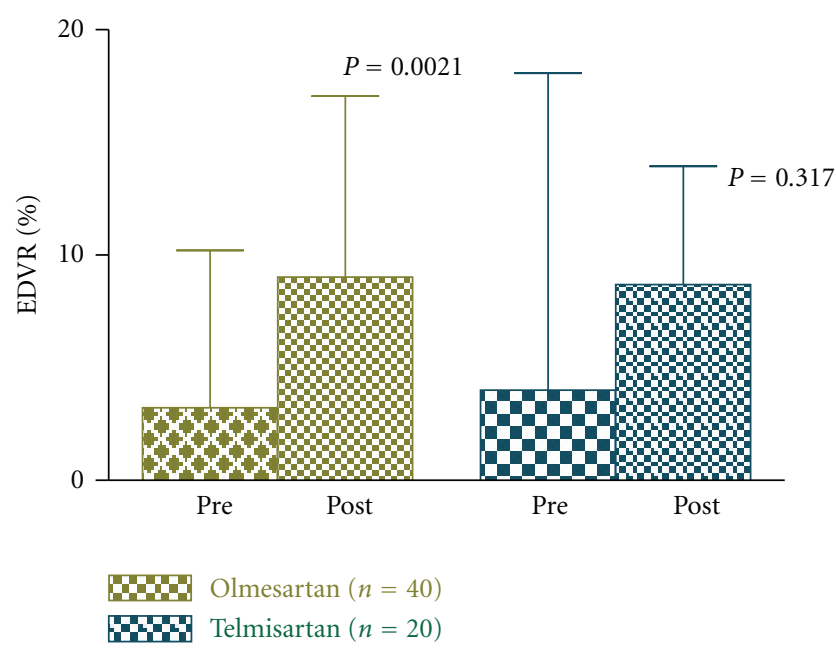

FIgURE 2: Effect of Olmesartan versus Telmisartan on Endothelial Dependent Vascular Response (EDVR \%) in Patents with Hypertension.

mild to moderate essential hypertensive patients, treatment with Telmisartan has the potential to improve PWV greater than effects due to lowering of BP alone. However, Uchida et al. [13] has shown that in essential hypertensive patients after 16 weeks of treatment with $10-40 \mathrm{mg}$ of Olmesartan cf PWV was reduced independently of the decrease in systolic BP. The mechanisms for such an independent improvement of arterial stiffness may possibly be improved by endothelial function and/or decreased oxidative stress. It is unlikely that structural changes would occur after 4 weeks of therapy.

We also observed an improvement in endothelial dependent vasodilator response to Salbutamol after with treatment both Olmesartan and Telmisartan. However the magnitude of improvement was more or less similar in both the treatments as compared to baseline. Our study results were in procession with the available literature; for example, change in postischemic reactive hyperemia as shown by Mediavilla and colleagues [14] was found to be greater after treatment

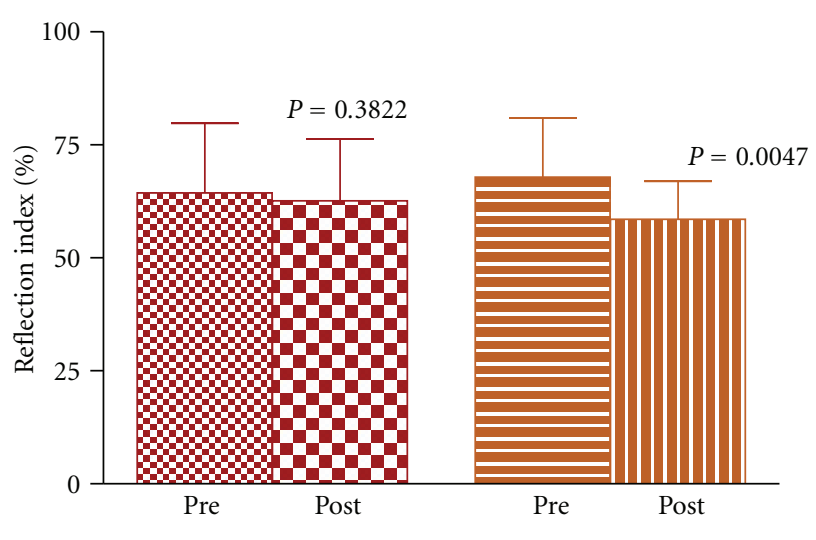

008 Olmesartan $(n=40)$
IIII Telmisartan $(n=20)$

FIGURE 3: Effect of Olmesartan versus Telmisartan on index of digital artery tone Reflection index (RI) in Patents with Hypertension.

with Telmisartan than Valsartan treatment, regardless of a similar reduction in BP exerted by both compounds. Tomiyama et al. [15] also demonstrated an improvement in endothelium-dependent coronary dilation in hypertensive patients after treatment with Olmesartan. Another possible benefit observed in our study population was reductions in serum MDA after treatment with both Olmesartan and Telmisartan. Such a finding was also observed in other studies such as Losartan, [16] Valsartan, [17] Irbesartan, [18] Olmesartan [19], and Telmisartan [20].

On comparison, Olmesartan showed a better improvement in cf PWV, EDVR, and MDA than Telmisartan. The magnitude of drop in supine either systolic or diastolic blood pressures was not significant statistically between the two groups. When we evaluated baseline demographic, biochemical, hemodynamic, vascular, and lipid peroxidation parameters, we found that they were similar in both Olmesartan and Telmisartan groups. One of the possible explanations for such an improvement with Olmesartan is that Olmesartan may have long lasting blood pressure lowering effects and vasculoprotective properties in patients with hypertension due to its strongest AT-II type 1 receptor antagonistic ability. In vitro studies have shown that the rank order of binding affinity to AT-II type 1 receptor varies between different ARBs and appears to be as follows: Valsartan $>$ Olmesartan $>$ Candesartan $>$ Irbesartan $>$ Telmisartan $>$ Losartan $>$ Eprosartan [21].

\section{Study Limitations}

In the present study, Olmesartan showed a better improvement in cf PWV, EDVR, and MDA than Telmisartan without much difference in $\mathrm{BP}$ reduction. However, such an interpretation needs carefulness because fewer numbers of patients received Telmisartan. The time at which they took the medication before each study day, concomitant medications and intrapatient variability can be confounding 
factors. Although, to confirm the role of free radicals in essential hypertension, we have estimated free radical marker MDA, we could not estimate concentration of serum nitric oxide or its end-product nitrites in the present study.

\section{Conclusion}

Both Olmesartan and Telmisartan, which are known to decrease blood pressures, have demonstrated their efficacy in reducing arterial stiffness. This was associated with an improvement in EDVR and a significant reduction of serum MDA levels in patients with essential hypertension. Olmesartan showed a better improvement in cf PWV, EDVR, and MDA than Telmisartan with an identical reduction in blood pressure. Thus, it can be concluded that, in patients with essential hypertension both the ARBs Olmesartan and Telmisartan offered their beneficial effects on cardiovascular system through multiple mechanisms.

\section{Conflict of Interests}

The authors declare that they have no conflict of interests.

\section{References}

[1] W. W. Nichols and M. O'Rourke, McDonald'S Blood Flow in arteries. Theoretical, Experimental and Clinical principles, Arnold E, London, UK, 4th edition, 1998.

[2] S. Kinlay, M. A. Creager, M. Fukumoto et al., "Endotheliumderived nitric oxide regulates arterial elasticity in human arteries in vivo," Hypertension, vol. 38, no. 5, pp. 1049-1053, 2001.

[3] J. A. Panza, A. A. Quyyumi, J. E. Brush, and S. E. Epstein, "Abnormal endothelium-dependent vascular relaxation in patients with essential hypertension," New England Journal of Medicine, vol. 323, no. 1, pp. 22-27, 1990.

[4] S. Laurent, "Arterial wall hypertrophy and stiffness in essential hypertensive patients," Hypertension, vol. 26, no. 2, pp. 355-362, 1995.

[5] T. W. Hansen, J. A. Staessen, C. Torp-Pedersen et al., "Prognostic value of aortic pulse wave velocity as index of arterial stiffness in the general population," Circulation, vol. 113, no. 5, pp. 664-670, 2006.

[6] R. M. Touyz, "Reactive oxygen species in vascular biology: role in arterial hypertension," Expert Review of Cardiovascular Therapy, vol. 1, no. 1, pp. 91-106, 2003.

[7] I. B. Wilkinson, A. Qasem, C. M. McEniery, D. J. Webb, A. P. Avolio, and J. R. Cockcroft, "Nitric oxide regulates local arterial distensibility in vivo," Circulation, vol. 105, no. 2, pp. 213-217, 2002.

[8] M. U. R. Naidu, B. M. Reddy, S. Yashmaina, A. N. Patnaik, and P. U. Rani, "Validity and reproducibility of arterial pulse wave velocity measurement using new device with oscillometric technique: a pilot study, BioMedical Engineering Online, vol. 4, article 49, 2005.

[9] S. C. Millasseau, F. G. Guigui, R. P. Kelly et al., "Noninvasive assessment of the digital volume pulse: comparison with the peripheral pressure pulse," Hypertension, vol. 36, no. 6, pp. 952-956, 2000.
[10] M. Dawes, P. J. Chowienczyk, and J. M. Ritter, "Effects of inhibition of the L-arginine/nitric oxide pathway on vasodilation caused by $\beta$-adrenergic agonists in human forearm," Circulation, vol. 95, no. 9, pp. 2293-2297, 1997.

[11] J. A. Beuge and S. D. Aust, “The thiobarbituric assay," Methods in Enzymology, vol. 52, p. 306, 1978.

[12] A. Mahmud and J. Feely, "Reduction in arterial stiffness with angiotensin II antagonist is comparable with and additive to ACE inhibition," American Journal of Hypertension, vol. 15, no. 4, pp. 321-325, 2002.

[13] H. Uchida, Y. Nakamura, M. Kaihara et al., "Efficiency of Telmisartan on morning home blood pressure and arterial wall stiffness in patients with mild hypertension," Hypertension Research, vol. 22, supplement 1, S148, p. 544, 2004.

[14] J. D. Mediavilla García, C. Fernández-Torres, F. Jaén Aguila, and J. Jiménez-Alonso, "Effect of olmesartan medoxomil on arterial stiffness in patients with essential hypertension," Medicina Clínica, vol. 128, no. 19, pp. 726-729, 2007.

[15] H. Tomiyama, J. Yamada, Y. Koji, K. Shiina, M. Yoshida, and A. Yamashina, "Effect of Telmisartan on forearm postischemic hyperemia and serum asymmetric dimethylarginine levels," American Journal of Hypertension, vol. 20, no. 12, pp. 1305-1311, 2007.

[16] M. Naya, T. Tsukamoto, K. Morita et al., "Olmesartan, but not amlodipine, improves endothelium-dependent coronary dilation in hypertensive patients," Journal of the American College of Cardiology, vol. 50, no. 12, pp. 1144-1149, 2007.

[17] S. Keidar, J. Attias, J. Smith, J. L. Breslow, and T. Hayek, “The Angiotensin-II receptor antagonist, losartan, inhibits LDL lipid peroxidation and atherosclerosis in apolipoprotein E-deficient mice," Biochemical and Biophysical Research Communications, vol. 236, no. 3, pp. 622-625, 1997.

[18] P. Dandona, V. Kumar, A. Aljada et al., "Angiotensin II receptor blocker valsartan suppresses reactive oxygen species generation in leukocytes, nuclear factor- $\kappa \mathrm{B}$, in mononuclear cells of normal subjects: evidence of an antiinflammatory action," Journal of Clinical Endocrinology and Metabolism, vol. 88, no. 9, pp. 4496-4501, 2003.

[19] S. Navalkar, S. Parthasarathy, N. Santanam, and B. V. Khan, "Irbesartan, an angiotensin type 1 receptor inhibitor, regulates markers of inflammation in patients with premature atherosclerosis," Journal of the American College of Cardiology, vol. 37, no. 2, pp. 440-444, 2001.

[20] S. I. Yamagishi, T. Matsui, K. Nakamura et al., "Olmesartan blocks inflammatory reactions in endothelial cells evoked by advanced glycation end products by suppressing generation of reactive oxygen species," Ophthalmic Research, vol. 40, no. 1, pp. 10-15, 2007.

[21] Y. Saitoh, W. Hongwei, H. Ueno, M. Mizuta, and M. Nakazato, "Telmisartan attenuates fatty-acid-induced oxidative stress and $\mathrm{NAD}(\mathrm{P}) \mathrm{H}$ oxidase activity in pancreatic $\beta$-cells," Diabetes and Metabolism, vol. 35, no. 5, pp. 392-397, 2009. 


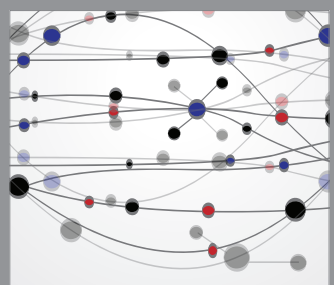

The Scientific World Journal
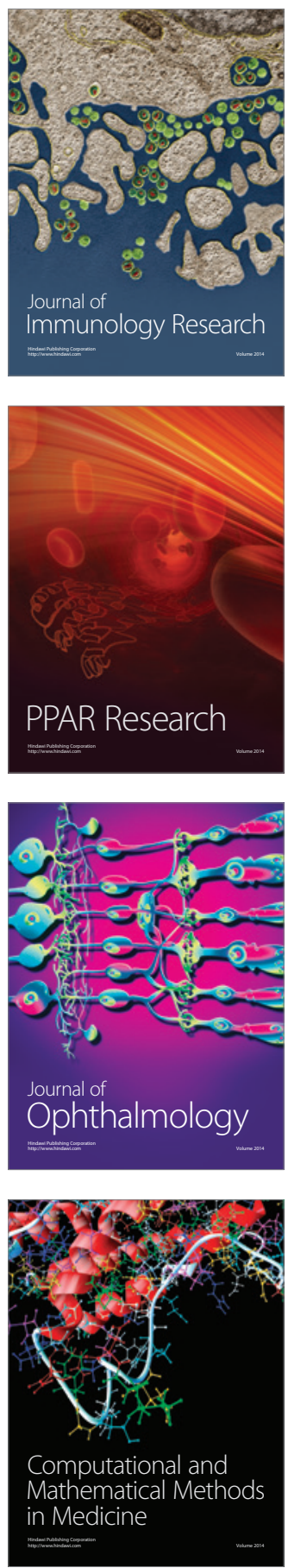

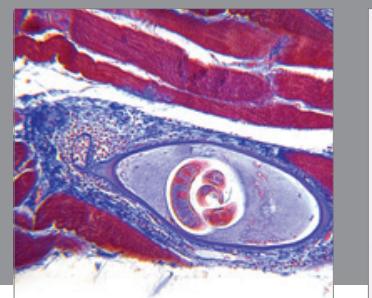

Gastroenterology

Research and Practice
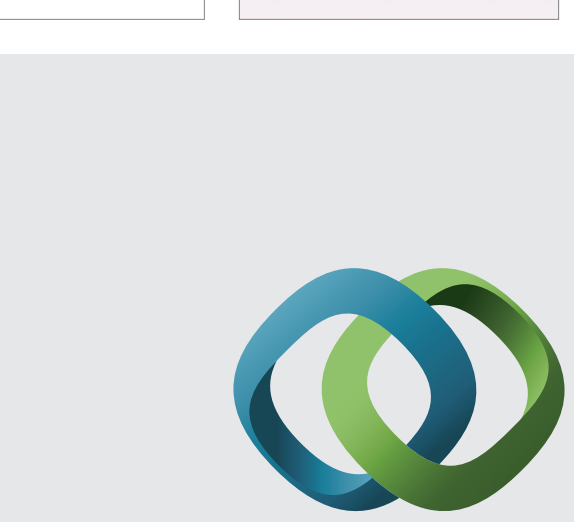

\section{Hindawi}

Submit your manuscripts at

http://www.hindawi.com
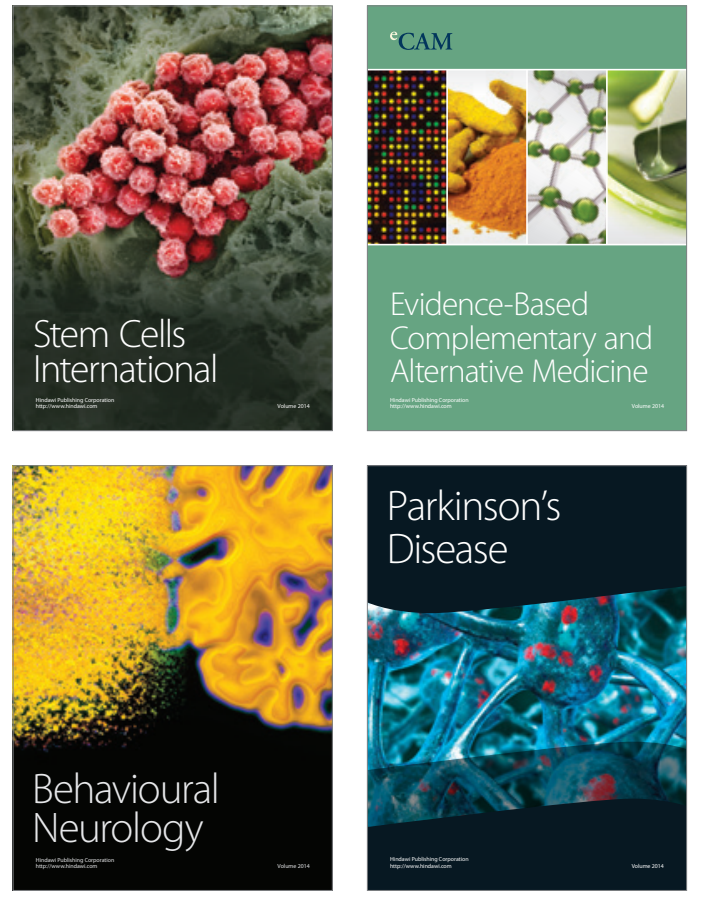
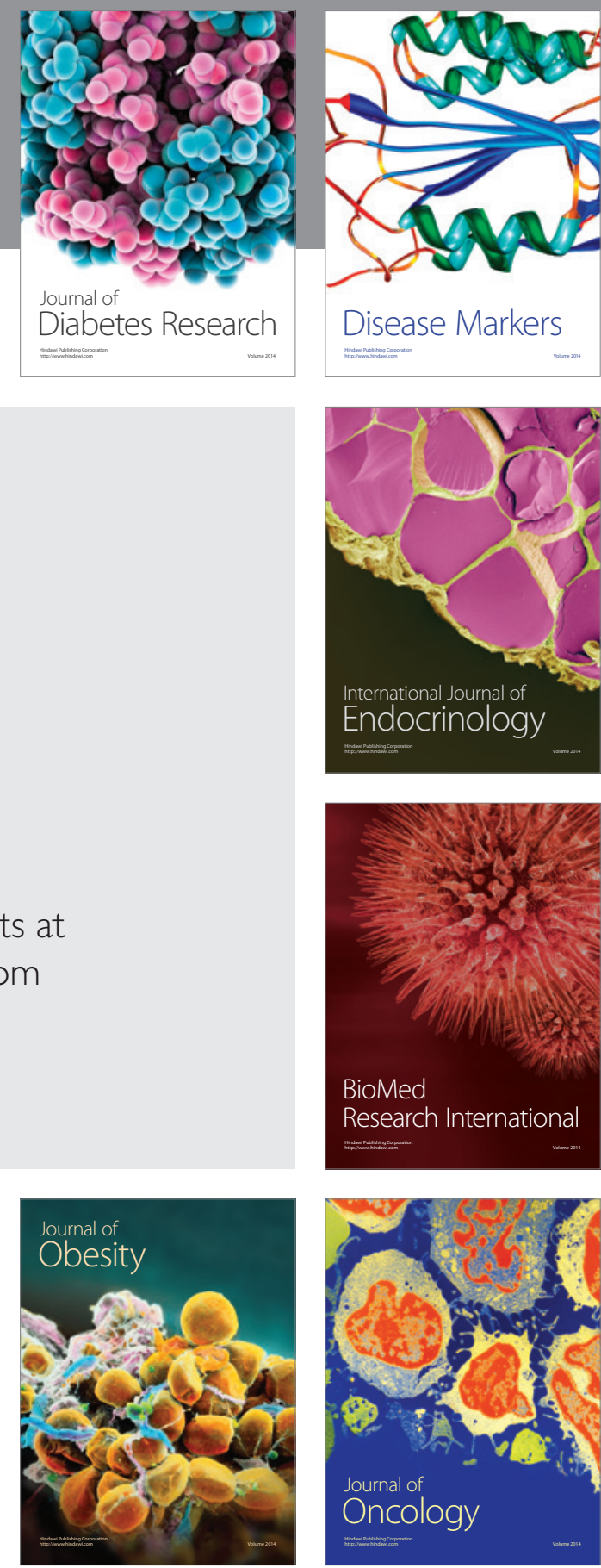

Disease Markers
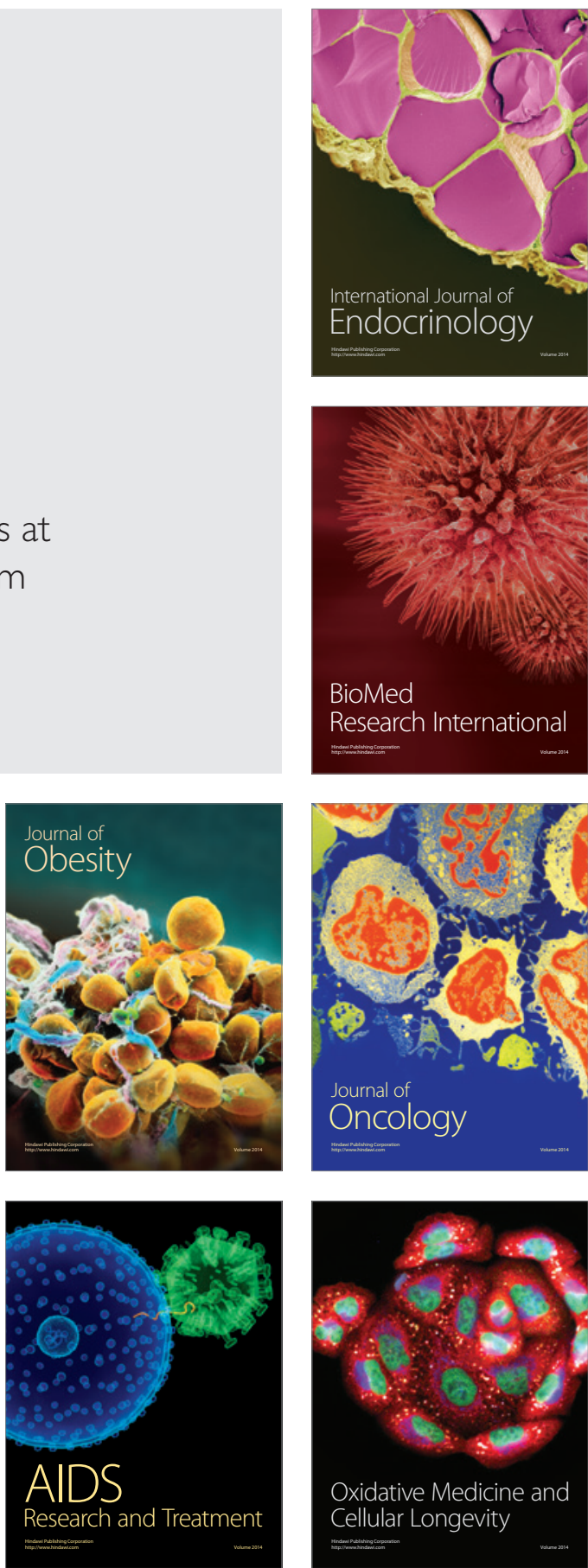